\title{
Pseudo-geographical representations of power system buses by multidimensional scaling
}

\author{
Florence Belmudes, Damien Ernst and Louis Wehenkel \\ Department of Electrical Engineering and Computer Science \\ University of Liège, Belgium \\ E-mails: \{florence.belmudes,dernst,l.wehenkel\}@ulg.ac.be
}

\begin{abstract}
Graphical representations of power systems are systematically used for planning and operation. The coordinate systems commonly used by Transmission System Operators are static and reflect the geographical positions of each equipment of the system. We propose in this work to position on a twodimensional map the different buses of a power system in a way such that their coordinates also highlight some other physical information related to them. These pseudo-geographical representations are computed by formulating multidimensional scaling problems which aim at mapping a distance matrix combining both geographical and physical information into a vector of two-dimensional bus coordinates. We illustrate through examples that these pseudo-geographical representations can help to gain insights into the power system physical properties.
\end{abstract}

Index Terms-power system visualization, multidimensional scaling, electrical distances

\section{INTRODUCTION}

Two-dimensional representations of power systems based on the geographical location of their devices have always been used for power system planning and operation. Originally, the - now universally-used - one-line diagrams were created as simplified representations of three-phase power systems. The need to incorporate in these diagrams some indications about the physical properties of the system quickly arose. Solutions using line widths were proposed to represent the power flows in the transmission lines [6]. Color shadings were used to represent limit violations. Also the availability status of the equipments was visualized, thanks to full or dotted lines.

As a matter of fact, a considerable effort was made over the last decade to enhance power system visualizations by exploiting available, both hardware and software, computational resources [7]. For example, color contours are now used to represent voltage magnitude variations across wide areas. Flows in transmission lines are visualized on pie charts, showing their loading, and this information can be supplemented by animated arrows showing the direction of the flows. These indicators are resized to reflect the state of the system. In parallel, solutions have recently been developed to match the one-line diagram of the system and the geographical information about the equipments, based on satellite views [5].

However, all of the currently used representations use static and mostly geographically based coordinate systems to locate the various devices of the power system in their twodimensional geometry.
We propose in this work a new methodology in order to enrich the existing two-dimensional graphical representations of power systems with any kind of useful information about their physical properties, in such a way that the locations of the buses of the system not only represent their geographical relation but also the variation of these physical properties. Our approach is based on using multidimensional scaling as a tool to transform composite distance measures combining both geographical and physical information about power system buses into two-dimensional coordinates. The physical information incorporated into these distance measurements can be diverse. As we will illustrate, they can for example represent the reduced impedance of the network between two buses, their electromechanical distance [2] or distances that quantify any kind of interesting physical information (like, e.g., nodal sensitivity factors) associated to these two buses.

Such "pseudo-geographical" representations can convey meanings, interpretations and knowledge on the power system, and therefore facilitate the planning and the operation of the system. As way of example, let us suppose that the distance between the buses on the map also represents the reduced impedance of the system between each pair of buses, and that the resulting map shows that the buses are located in two main zones which are rather distant from each other. This can be an indication that low frequency oscillations may occur between these two zones of the system and that specific devices should be installed to modulate the power transfer between these zones (e.g., Thyristor Controlled Series Capacitor).

To compute the pseudo-geographical coordinates, we rely technically on multi-dimensional scaling methods which are often used in information visualization for exploring similarities or dissimilarities in high-dimensional datasets [3]. We have adapted these methods to be able to control the tradeoff between the fidelity of representing the geographical and the physical information.

The rest of this paper is organized as follows. Section II describes the optimization problem lying behind the construction of these pseudo-geographical representations of power system buses. Two concrete application cases are introduced in Section III. In Section IV, an algorithm is proposed to solve the optimization problem previously described. Section V presents the representations obtained for the two illustration cases and conclusions are drawn in Section VI. 


\section{Problem Statement}

The first step in the construction of a pseudo-geographical representation of the buses of a power system is the choice of the data to represent. For this task, the reader can refer to the illustrations provided in Section III. The chosen data have to be expressed as distances between the buses of the system. If we consider a power system with $n$ buses, the input data consist of a set of $\frac{n(n-1)}{2}$ distances, one distance being defined for each pair of buses. For the sake of simplicity, we suppose that these distances are represented in a distance matrix format. This matrix, denoted by $D$, is a symmetric $n$ by- $n$ matrix of real non-negative elements. An element $d_{i j}$ of matrix $D$ corresponds to the distance between buses $i$ and $j$. The diagonal terms of this matrix are identically null since the distance between one bus and itself is equal to zero.

Given this matrix of pairwise distances, it is possible to compute a set of two-dimensional coordinates $\left\{\left(x_{k}, y_{k}\right)\right\}_{k=1}^{n}$ for the buses such that the Euclidean distances between these coordinates approximate the distances given in matrix $D$. This can be done by solving the following optimization problem:

$$
\begin{array}{r}
\underset{\left(x_{1}, y_{1}\right), \ldots,\left(x_{n}, y_{n}\right)}{\arg \min } \sum_{i=1}^{n} \sum_{j=i+1}^{n}\left(\sqrt{\left(x_{i}-x_{j}\right)^{2}+\left(y_{i}-y_{j}\right)^{2}}\right. \\
\left.-d_{i j}\right)^{2} .
\end{array}
$$

To the set of node coordinates which is solution of this optimization problem, corresponds a pseudo-geographical representation of the buses of the power system. There are several things to comment about the problem expressed in Equation (1). First, the objective function is always positive and, generally, even strictly positive. In the particular case where the matrix $D$ gathers the geographical distances between buses, its minimum is however equal to zero. The solution of (1) is also non-unique. Indeed, any pseudo-geographical map obtained by translating or rotating the pseudo-geographical map computed when solving (1) is also solution of (1) since such transformations leave the interbus distances unchanged.

Because we are not really interested to have a pseudogeographical map that represents the distances given in $D$ but well the ratio between these distances, we consider that any pseudo-geographical map obtained by scaling a solution of (1) is also as good as a solution of (1). Among the (infinite size) set of pseudo-geographical maps defined by applying any combination of translation, rotation and scaling operators to a solution of (1), we have decided to select the one in which the pseudo-geographical coordinates of two particular buses - defined a priori and referred to as reference buses coincide with their geographical coordinates. Deciding to have their position fixed in the pseudo-geographical representation provides the user with key for efficient interpretation.

Later in Section IV-B, we will carefully describe the similarity transformation (which is a composition of a translation, a rotation and a homothety) to use to make the pseudogeographical position of the two reference buses coincide with their geographical location.
Even with such a calibration, the resulting representation may be so different from the geographical one that the users may have difficulties to interpret the obtained map. In such cases, it would be more desirable to have a map corresponding to a suboptimal solution of (1) but which looks more alike the geographical map. To address this problem, we propose to embed the geographical bus coordinates in the input data of problem (1). To do so, we suggest to replace the $d_{i j}$ terms that appear in Equation (1) by terms expressing a "mixed" distance, denoted by $d_{i j}^{m}$, formed by taking a convex linear combination of the distance $d_{i j}$ and the geographical distance between buses $i$ and $j$. If we denote by $d_{i j}^{g e o}$ this geographical distance, the mixed distance can be formulated as:

$$
d_{i j}^{m}=\lambda d_{i j}+(1-\lambda) d_{i j}^{g e o},
$$

where the parameter $\lambda \in[0,1]$. The value assigned to this parameter reflects the importance that is given to the distances $d_{i j}$ with respect to the distances $d_{i j}^{g e o}$.

The optimization problem lying behind the computation of a representation based on these mixed distances writes:

$$
\begin{array}{r}
\underset{\left(x_{1}, y_{1}\right), \ldots,\left(x_{n}, y_{n}\right)}{\arg \min _{i=1}} \sum_{j=i+1}^{n} \sum^{n}\left(\sqrt{\left(x_{i}-x_{j}\right)^{2}+\left(y_{i}-y_{j}\right)^{2}}\right. \\
\left.-\left(\lambda d_{i j}+(1-\lambda) d_{i j}^{g e o}\right)\right)^{2} .
\end{array}
$$

If we set $\lambda=0$, the coordinates of the buses will only depend on their geographical distances and, among all solutions of the argmin problem, there will be the set of geographical coordinates of the buses. To the contrary, if $\lambda$ is set equal to 1 , the interbus geographical distances will not be taken into account, and, as a consequence, the pseudo-geographical map might be difficult to interpret. When $\lambda$ increases within $[0,1]$, the relative weight of the data contained in matrix $D$ with respect to the geographical distances between buses in the computed representation also increases.

\section{EXAMPLES OF APPLICATION CASES}

We give in this section two concrete examples of data on which we will illustrate our approach for creating pseudogeographical representations.

\section{A. Visualizing the reduced impedances between buses}

The first set of data is the set of the reduced admittances between each pair of buses of the system. The reduced impedance between two buses is obtained by reducing the admittance matrix of the network to these two buses, and by computing the modulus of the inverse of this value.

For a power system with $n$ buses, this procedure yields $\frac{n(n-1)}{2}$ values (one for each pair of buses) from which a distance matrix $D$ can be built. These reduced impedances can be seen as electrical distances between buses since they provide a good image of "how distant" two buses are on an electrical point of view. 
The electrical distance between two buses reflects for instance how likely their voltage angles are close or how a shortcircuit at a specific bus will affect the currents arriving at the other buses. Visualizing this information can certainly help to get better insights into the power system physics.

\section{B. Visualizing the voltage sensitivities of the buses}

For this second example, we choose to work with voltage variations from static security analyses. The variations are computed by considering always the same base case configuration and by running a power flow to compute the voltage drops induced by the loss of a generator. If we assume that the generators are numbered from 1 to $n_{g}$ and if we denote by $\Delta V_{i}^{g}$ the variation of the voltage magnitude at bus $i$ when generator $g$ is lost, we can associate to each bus a vector $\Delta V_{i}=\left(\Delta V_{i}^{g}\right)_{g=1}^{n_{g}}$ collecting its voltage variations.

In order to create a pseudo-geographical representation of these voltage sensitivities, the information contained in vectors $\Delta V_{i}$ has to be converted into interbus distances. On this purpose, we compute the Euclidean distance between these "voltage variation vectors". The distance between buses $i$ and $j$ is thus set equal to $: d_{i j}=\sqrt{\sum_{g=1}^{n_{g}}\left(\Delta V_{i}^{g}-\Delta V_{j}^{g}\right)^{2}}$.

These distance measurements express the dissimilarities between the voltage variations at the different buses after a loss of generation.

\section{Computational MEthod}

We describe in this section our approach for solving the optimization problem given by Equation (3) (see Section II). We first develop the algorithm used to compute a solution this problem. Afterwards, we carefully detail the similarity transformation to apply to this solution in order to make the pseudo-geographical position of the two reference buses coincide with their geographical location.

\section{A. Resolution of the optimization problem}

The first stage of approach is based on an optimization algorithm borrowed from the multidimensional scaling (MDS) literature. This algorithm is known as the SMACOF algorithm [4] (the acronym SMACOF stands for "Scaling by Majorizing a Complicated Function"). It will be explained at the end of this section after having introduced the mathematical background on which it relies.

Let us denote by $X^{\text {geo }} \in \mathbb{R}^{n \times 2}$ the matrix of the geographical coordinates of the buses, by $D \in \mathbb{R}^{n \times n}$ the matrix containing the interbus distance measurements and by $X \in \mathbb{R}^{n \times 2}$ the set of pseudo-geographical coordinates we want to compute.

The problem (3) can equivalently be written as:

$$
\underset{X}{\arg \min } f(X)
$$

where

$$
f(X)=\sum_{i=1}^{n} \sum_{j=i+1}^{n}\left(\sqrt{\sum_{k=1}^{2}\left(x_{i k}-x_{j k}\right)^{2}}-d_{i j}^{m}\right)^{2} .
$$

The function $f$ defined in Equation (5) has the form of the classical stress function one commonly seeks to minimize in an MDS problem. It can be expanded as follows:

$$
\begin{array}{r}
f(X)=\sum_{i=1}^{n} \sum_{j=i+1}^{n} \sum_{k=1}^{2}\left(x_{i k}-x_{j k}\right)^{2}+\sum_{i=1}^{n} \sum_{j=i+1}^{n}\left(d_{i j}^{m}\right)^{2} \\
-\sum_{i=1}^{n} \sum_{j=i+1}^{n}\left(\sqrt{\sum_{k=1}^{2}\left(x_{i k}-x_{j k}\right)^{2}}\right) d_{i j}^{m} .
\end{array}
$$

The first term of this sum can also be written:

$$
\sum_{i=1}^{n} \sum_{j=i+1}^{n} \sum_{k=1}^{2}\left(x_{i k}-x_{j k}\right)^{2}=\operatorname{tr} X^{\prime} A X
$$

with $A \in \mathbb{R}^{n \times n}$ being such that $a_{i i}=n-1$ and $a_{i j}=a_{j i}=$ -1 .

The second term of $f(X)$ does not depend on $X$ and can be seen as a constant, so we set:

$$
k_{0}=\sum_{i=1}^{n} \sum_{j=i+1}^{n}\left(d_{i j}^{m}\right)^{2} .
$$

In the third term of $f(X)$, we denote by $\operatorname{dist}_{i, j}(X)$ the Euclidean distance between buses $i$ and $j$ :

$$
\sqrt{\sum_{k=1}^{2}\left(x_{i k}-x_{j k}\right)^{2}}=\operatorname{dist}_{i, j}(X) .
$$

Given (7), (8) and (9), Equation (6) can be written concisely as:

$$
f(X)=\operatorname{tr} X^{\prime} A X+k_{0}-2 \sum_{i=1}^{n} \sum_{j=i+1}^{n} \operatorname{dist}_{i, j}(X) d_{i j}^{m} .
$$

The third term of this expression is non-convex and makes the resolution of the problem (4) difficult. To address this problem, one can majorize this term by a convex expression to get a new objective function, easier to minimize. The SMACOF algorithm exploits the following majorization, based on the Cauchy-Schwartz inequality:

$$
\begin{aligned}
\sum_{k=1}^{2}\left(x_{i k}-x_{j k}\right)\left(y_{i k}-y_{j k}\right) \leq & \left(\sum_{k=1}^{2}\left(x_{i k}-x_{j k}\right)^{2}\right)^{1 / 2} \\
& \times\left(\sum_{k=1}^{2}\left(y_{i k}-y_{j k}\right)^{2}\right)^{1 / 2} \\
\leq & \operatorname{dist}_{i j}(X) \operatorname{dist}_{i j}(Y),
\end{aligned}
$$

where $Y \in \mathbb{R}^{n \times 2}$ can be interpreted as another set of coordinates for the buses.

If we multiply both sides of the inequality by $(-1)$ and divide by $\operatorname{dist}_{i j}(Y)$, we obtain:

$$
-\operatorname{dist}_{i j}(X) \leq \frac{\sum_{k=1}^{2}\left(x_{i k}-x_{j k}\right)\left(y_{i k}-y_{j k}\right)}{\operatorname{dist}_{i j}(Y)} .
$$


By summing over $i=1 \ldots n$ and $j=i+1 \ldots n$ we obtain the majorizing expression:

$$
\begin{aligned}
-2 \sum_{i=1}^{n} \sum_{j=i+1}^{n} \operatorname{dist}_{i, j}(X) d_{i j} \leq & -2 \sum_{i=1}^{n} \sum_{j=i+1}^{n} \sum_{k=1}^{2} \frac{d_{i j}^{m}}{\operatorname{dist}_{i j}(Y)} \\
& \times\left(x_{i k}-x_{j k}\right)\left(y_{i k}-y_{j k}\right) \\
\leq & -2 \operatorname{tr} X^{\prime} B(Y) Y,
\end{aligned}
$$

with $B(Y) \in \mathbb{R}^{n \times n}$ being such that:

$$
\begin{aligned}
& b_{i j}=\left\{\begin{array}{c}
-\frac{d_{i j}^{m}}{\operatorname{dist}_{i j}(Y)} \text { for } i \neq j \text { and } \operatorname{dist}_{i j}(Y) \neq 0 \\
0 \quad \text { for } i \neq j \text { and } \operatorname{dist}_{i j}(Y)=0
\end{array}\right. \\
& b_{i i}=\sum_{j=1, j \neq i}^{n} b_{i j} .
\end{aligned}
$$

By combining (10) and (13), the function $f$ itself can be majorized:

$$
f(X) \leq \operatorname{tr} X^{\prime} A X+k_{0}-2 \operatorname{tr} X^{\prime} B(Y) Y=g(X) .
$$

The function $g$ is a quadratic function of $X$. The minimum of the function $g$ is obtained when its derivative is equal to zero, i.e.:

$$
\nabla g(X)=2 A X-2 B(Y) Y=0 .
$$

The value of $X$ minimizing $g(X)$ is such that:

$$
A X=B(Y) Y .
$$

As the inverse $A^{-1}$ does not exist since $A$ is not full rank, this linear equation in $X$ cannot be solved by premultiplying both sides of (17) by $A^{-1}$. The Moore-Penrose inverse, given by $A^{+}=\left(A+\mathbf{1}_{n, n}\right)^{-1}-n^{-2} \mathbf{1}_{n, n}$ (where $\mathbf{1}_{n, n}$ is the matrix such that $\left.\mathbf{1}_{n, n}(i, j)=1 \forall(i, j) \in\{1, \ldots, n\}^{2}\right)$, is used in the SMACOF algorithm. The matrix $X$ minimizing $g(X)$, and subsequently $f(X)$, is the following:

$$
X=A^{+} B(Y) Y .
$$

It can be shown that the solution computed from (18) is such that $f(X) \leq f(Y)$. The SMACOF algorithm exploits this property to iteratively compute solutions with decreasing values of $f$. The solution computed at iteration $i$, denoted by $X_{i}$, is equal to $A^{+} B\left(X_{i-1}\right) X_{i-1}$. The tabular version of the procedure used in our simulations is given in Figure 1.

\section{B. Geometrical transformation}

In our simulation results, the coordinates $X^{M D S}$ computed by the SMACOF algorithm will not be used as such to plot the data. To these coordinates will be applied successively a translation, a rotation and a homothetic transformation to have two buses matching their geographical position (see Section II). These transformations are detailed on Figure 2 and are further illustrated on an example in Figure 3.

On this latter figure, the four red crosses correspond to the geographical location of these points, and the four blue circles to their MDS location as outputted by algorithm given
Problem definition: an $n$-by- $n$ distance matrix, a matrix $X^{g e o} \in \mathbb{R}^{n \times 2}$ of geographical coordinates.

Algorithm parameters: a small positive value $\epsilon$, which is the minimum decrease of $f$ after an iteration for not stopping the iterative process, and a maximal number of iterations IterMax.

Output: a matrix $X^{M D S} \in \mathbb{R}^{n \times 2}$ of pseudo-geographical coordinates.

\section{Algorithm:}

Step 1. Set $X_{0}=X^{g e o}$.

Set iteration counter Iter $=0$.

Step 2. Compute $f_{0}=f\left(X_{0}\right)$. Set $f_{-1}=f_{0}$.

Step 3. While Iter $=0$ or $\left(f_{\text {Iter }-1}-f_{\text {Iter }}\right)>\epsilon$ and Iter $\leq$ IterMax) do:

Set Iter $\leftarrow$ Iter +1 .

Compute $B\left(X_{\text {Iter }-1}\right)$ by using Equation (14).

Set $X_{\text {Iter }}=A^{+} B\left(X_{\text {Iter }-1}\right) X_{\text {Iter }-1}$.

Compute $f_{\text {Iter }}=f\left(X_{\text {Iter }}\right)$.

Set $X_{\text {Iter }+1}=X_{\text {Iter }}$.

Step 4. Set $X^{M D S}=X_{\text {Iter }}$. Output $X^{M D S}$.

Fig. 1. A tabular version of the SMACOF algorithm for solving problem (4).

Problem definition: the coordinates $X^{M D S} \in \mathbb{R}^{n \times 2}$ computed by the algorithm given on Figure 1, the positions of the two reference buses on the geographical map. These positions are denoted by $B_{r 1}^{g e o}$ and $B_{r 2}^{g e o} . B_{r 1}^{M D S}$ and $B_{r 2}^{M D S}$ refer to their position defined by $X^{M D S}$.

Output: a set of coordinates $X$ such that the reference buses are positioned as in the geographical map.

\section{Algorithm:}

Step 1. Translate $X^{M D S}$ along vector $v$ such that $v=\overrightarrow{B_{r 1}^{M D S} B_{r 1}^{g e o}}$.

Step 2. Apply to the resulting map a rotation around $B_{r 1}^{g e o}$ of angle $\theta=\operatorname{angle}\left(\overrightarrow{B_{r 1}^{M D S} B_{r 2}^{M D S}}, \overrightarrow{B_{r 1}^{g e o} B_{r 2}^{g e o}}\right)$.

Step 3. Apply to the resulting map a homothetic transformation of origin $B_{r 1}^{g e o}$ and of dilatation factor $k=\frac{\text { length }\left(B_{r 1}^{g e o} B_{r 2}^{g e o}\right)}{\text { length }\left(B_{r 1}^{M D S} B_{r 2}^{M D S}\right)}$.

Fig. 2. A fully specified algorithm for transforming the coordinates outputted by the multidimensional scaling algorithm so that the position of the reference buses $B_{r 1}^{M D S}$ and $B_{r 2}^{M D S}$ coincide with their geographical location.

in Figure 1. We apply successively the translation, rotation and homothetic operators defined on Figure 2 to the blue circles, in order to make the blue circles 1 and 3 coincide with the 1 and 3 red crosses.

Figure 3 shows the initial representations as well as those obtained after every stage of the similarity transformation. 


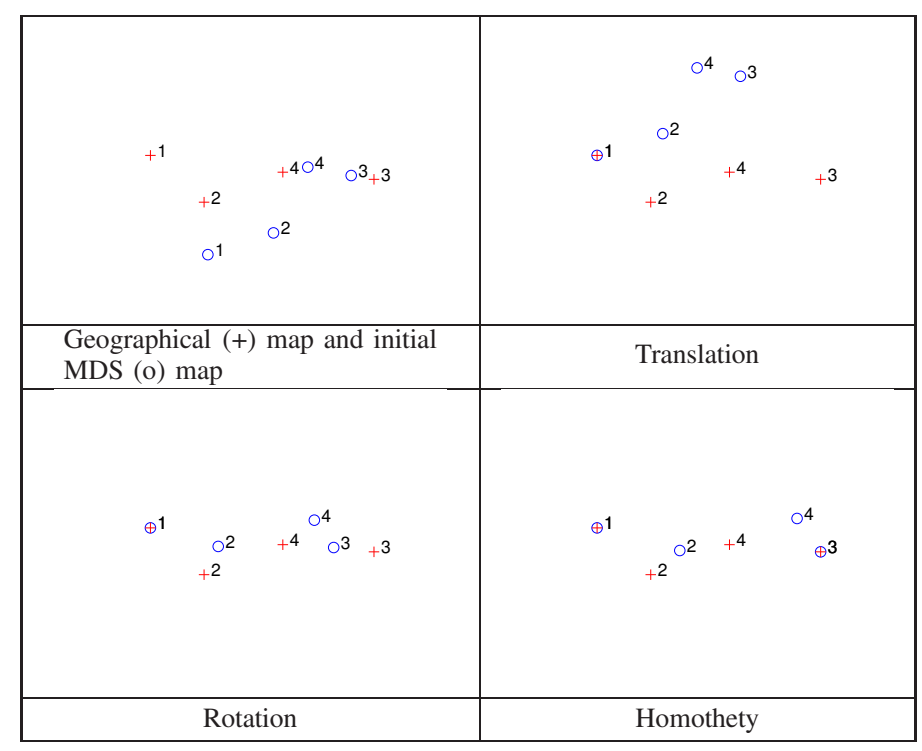

Fig. 3. Illustration of the similarity transformation (successive translation, rotation and homothety) for generating, from a set of coordinates computed by the SMACOF algorithm, a set of pseudo-geographical coordinates such that the points 1 and 3 of the pseudo-geographical map are positioned as in the geographical map.

\section{ILLUSTRATIONS}

We illustrate in this section our approach for computing pseudo-geographical representations of the two application cases introduced in Section III. The benchmark power system considered in this section is the IEEE 14 bus system [1], which has been vastly used in the literature as a test problem. Its one-line diagram is shown in Figure 4. It is composed of five synchronous machines: two generation units are located at buses 1 and 2 respectively, and the three other machines, connected to buses 3, 6 and 8 , are synchronous compensators used only for reactive power supply. The 20 transmission lines of the system are either at $132 \mathrm{kV}$ or $33 \mathrm{kV}$, the $33 \mathrm{kV}$ part of the network being located at the top of Figure 4 and the transformers at buses 4,5 and 7 . The total amount of load is about $259 \mathrm{MW}$.

\section{A. Pseudo-geographical representation of the reduced impedances between buses}

Figure 5 reports the pseudo-geographical representations of the reduced impedances between the buses of IEEE 14 bus test system, for different values of the parameter $\lambda$. The case $\lambda=0$ corresponds to the classical geographical representation of this network. The case $\lambda=0.95$ corresponds to the representation of the buses according to a mixed distance as defined in (2). The case $\lambda=1$ is a representation exclusively based on the electrical distances between buses.

Obviously, the geographical positions of the buses do not reflect their electrical distances. For example, buses 1, 2, 4 and 5 are much closer electrically than they are geographically. It is also worth noticing that bus 8 , which is connected to buses 4 and 9 through a three windings transformer, appears quite close to these buses on the geographical representation while

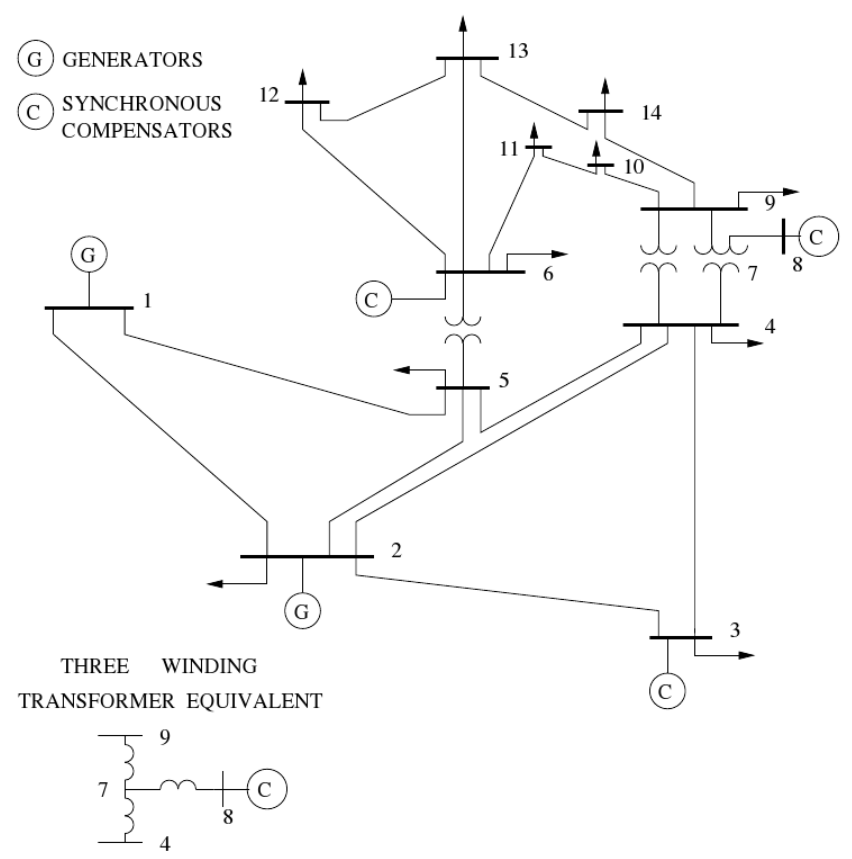

Fig. 4. IEEE 14 bus system

it is not the case on the pseudo-geographical ones. This was expected since the windings of a transformer generally have a rather high reactance whose value is in the range of the reactance value of a few tens of kilometers long transmission line. Similarly, we observe that the buses in the upper part of the system, which correspond to the lower voltage level (33 $\mathrm{kV}$ ), are more remotely located when taking into account their electrical distances.

\section{B. Pseudo-geographical representation of the voltage sensi- tivities of the buses}

Figure 6 illustrates the results of our experiments when considering the voltage sensitivities of the buses of IEEE 14 bus test system. As in the previous example, the three subfigures correspond to three increasing values of parameter $\lambda(0,0.8$ and 1 respectively).

These figures clearly show that for large values of $\lambda$, the pseudo-geographical representations considerably differ from the pure geographical one.

We notice that the system has five synchronous machines controlling the voltage, which are connected to buses 1,2 , 3,6 and 8. Since these buses are controlled in voltage except when considering the loss of the generator which is connected to them, their sensitivities are quite small and similar. It is therefore not surprising to see that they all appear next to each other on the map when $\lambda=1$. Another interesting observation about this map is the far remote location of bus 4 with respect to the other elements of the system. This shows that the voltage variations caused by the loss of generation at this bus are different from the ones at the other buses. Notice that, by plotting the voltage profiles, we have observed that whatever 


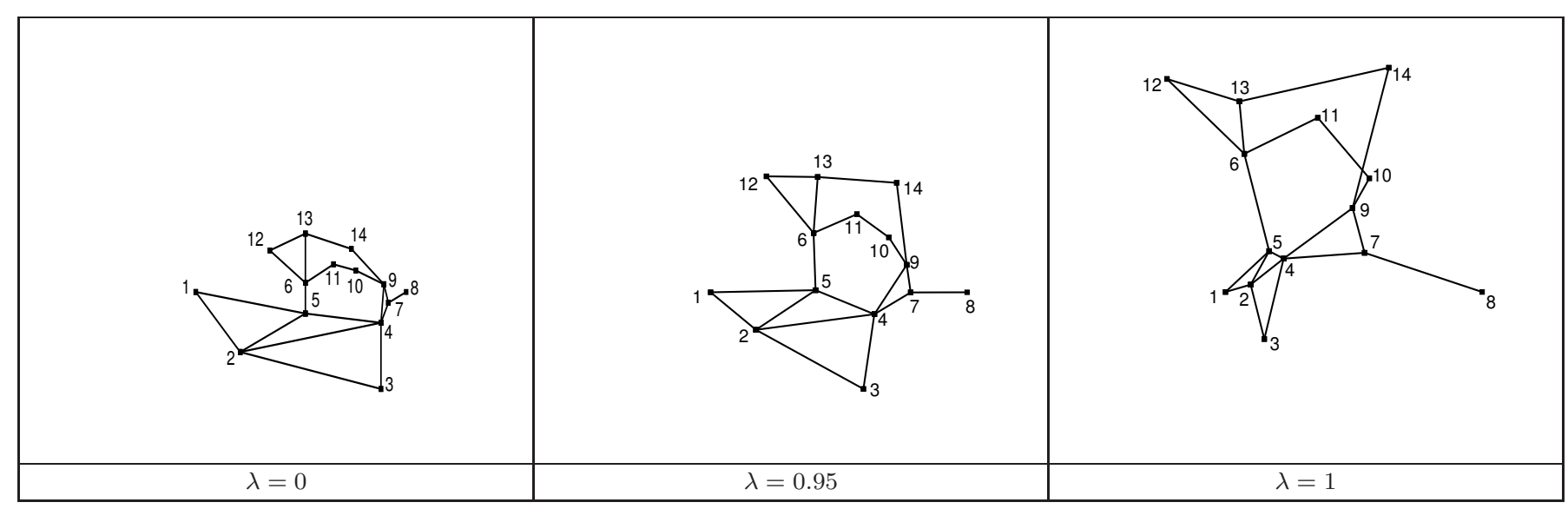

Fig. 5. Pseudo-geographical representations of the IEEE 14 bus system based on the reduced impedances. Buses 1 and 8 are taken as reference buses.

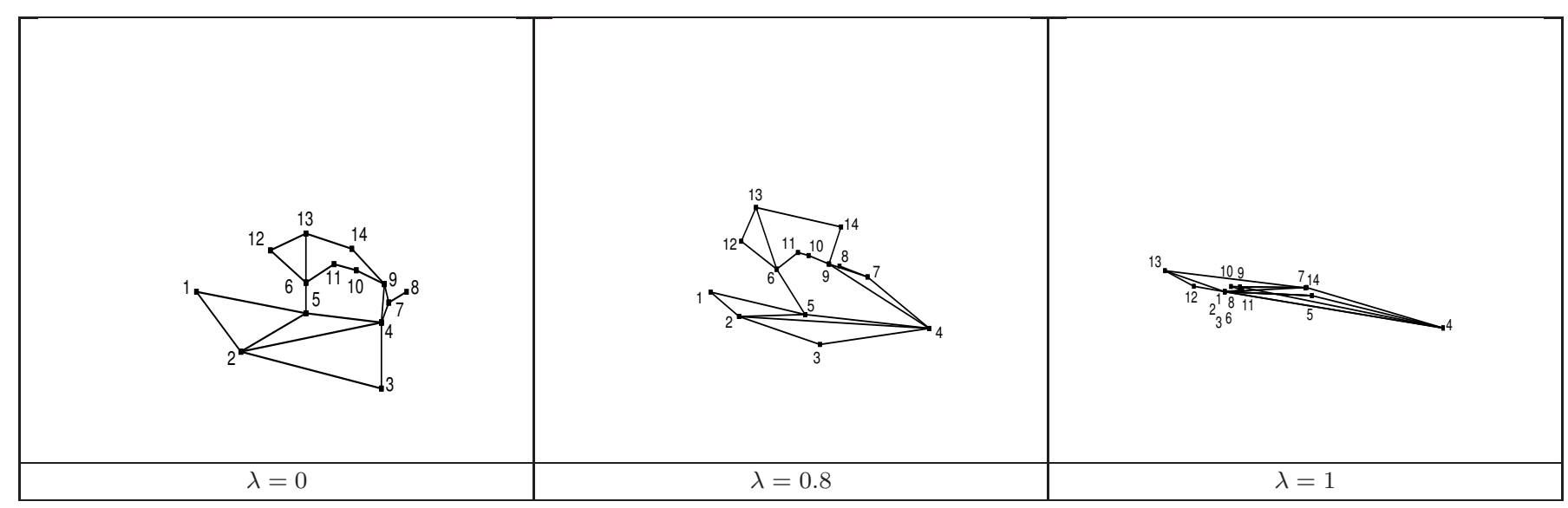

Fig. 6. Pseudo-geographical representations of the IEEE 14 bus system based on the voltage sensitivities. Buses 1 and 4 are taken as reference buses.

the generator lost, the voltage at bus 4 was the lowest of the bus voltages. Conversely, the bus 13 is located on the far other end of the representation; it turns out that this bus, which is located in the low voltage part of the system, experiences also strong voltage variations but which are de-correlated to those of bus 4 .

\section{CONCLUSION}

We have proposed in this paper a new approach for visualizing power system data that can be expressed as pseudodistances between buses of the system. The approach works by processing these data with a multi-dimensional scaling algorithm to obtain a two-dimensional map where the different buses are positioned in a way that the distance between every two buses represents these pseudo-distances. The approach has been illustrated on two case studies demonstrating the interest of mixing geographical and physical information to design power system representations. The results have shown that these pseudo-geographical representations can complement existing visualization tools for planning and operation of a power system.

\section{ACKNOWLEDGMENT}

Florence Belmudes thanks the FRIA (French acronym for Belgian Fund for Research in Industry and Agriculture) for supporting her research.
Damien Ernst is a Research Associate of the Belgian FNRS (French acronym for National Fund of Scientific Research) from which he acknowledges the financial support.

This paper presents research results of the Belgian Network DYSCO, funded by the Interuniversity Attraction Poles Programme, initiated by the Belgian State, Science Policy Office. The scientific responsibility rests with its authors.

\section{REFERENCES}

[1] IEEE 14 bus test system: available at http://www.ee.washington.edu/ research/pstca/pf14/pg_tca14bus.htm.

[2] R. Belhomme and M. Pavella. A composite electromechanical distance approach to transient stability. IEEE Transactions on Power Systems, 6:622-631, 1991.

[3] I. Borg and P. Groenen. Modern Mutlidimensional Scaling: Theory and Applications. Springer New York, 2005.

[4] J. de Leeuw. Applications of Convex Analysis to Multidimensional Scaling. In J.R. Barra, F. Brodeau, G. Romier, and B. Van Cutsem, editors, Recent Developments in Statistics, pages 133-146. North Holland Publishing Company, Amsterdam, 1977.

[5] F.B. Lemos and F. Kober. Experience on development and utilization of Google ${ }^{\mathrm{TM}}$ Earth in a DMS environment. Abstract presented at the 10th International Workshop on Electric Power Control Centers (EPCC), 2009.

[6] P.M. Mahadev and R.D. Christie. Envisioning power system data: concepts and a prototype system state representation. IEEE Transactions on Power Systems, 8(3):1084-90, August 1993.

[7] T.J. Overbye and D.A. Wiegmann. Reducing the risk of major blackouts through improved power system visualization. In Proc. 2005 Power Systems Computational Conference (PSCC), Liège, Belgium, August 2005 